\title{
Controlador digital predictivo de demanda máxima de potencia eléctrica
}

\author{
Juan Manuel Ojeda Sarmiento \\ Southern Perú \\ Ingeniería Industrial n. 28, 2010, ISSN 1025-9929, pp 111-137 \\ Recibido: 20 de mayo del 2010 / Aprobado: 12 de julio del 2010
}

\begin{abstract}
REsumen: En este artículo se describen el diseño y la construcción del controlador digital predictivo de máxima demanda de potencia eléctrica. El dispositivo gestiona cargas de cualquier planta, conectando y desconectando grupos de equipos, en intervalos de tiempo, sobre la base de prioridades del negocio y considerando la estructura tarifaria. Está compuesto por un algoritmo de control, un bloque lógico que establece la secuencia de apagado y encendido de grupos de cargas, un modelo matemático que ordena estos grupos para que operen en ciertas franjas tarifarias y un módulo de pronóstico de demanda, basado en redes neuronales artificiales para anticiparse a la presencia de posibles demandas no deseadas.
\end{abstract}

Palabras clave: gestión de carga eléctrica / predicción de demanda / redes neuronales artificiales

\section{Design and construction of a predictive maximum demand controller}

\begin{abstract}
This paper describes the design and construction of the digital predictive controller of peak electrical demand. The device is able to avoid unnecessary peak demands, plugging and unplugging computer groups during time intervals, based on business priorities and considering the tariff structure. It's composed of a control algorithm, a logic block that provides the off-on sequence of load groups, a mathematical model that orders these groups to operate in some hours as well as a demand forecasting module based on neural networks artificial to anticipate the possible presence of unwanted demand source.
\end{abstract}

Keywords: load management / demand forecasting / artificial neural networks 


\section{INTRODUCCIÓN}

El consumo final de energía eléctrica del sector industrial en el país, incluyendo las mypes, llegó a $8.518 \mathrm{GWh}$ durante el 2008, lo que representa el $28,66 \%$ del consumo total de energía eléctrica del país, según datos del Ministerio de Energía y Minas (Minem 2009); sin embargo, las empresas de este sector presentan importantes oportunidades de ahorro.

En el rubro textil, por ejemplo, se han observado oportunidades de ahorro del orden de 4 a $11 \%$ en promedio, según una evaluación efectuada en mayo del 2008 por la Dirección General de Electricidad. Estas oportunidades de ahorro varían de acuerdo con el tamaño de la instalación, las características del proceso y las políticas de gestión energética de la empresa. El reto consiste en aprovechar esas oportunidades de ahorro, desarrollando tecnologías innovadoras con bajo costo de implementación, que puedan extenderse masivamente a las mypes para reducir sus sobrecostos de facturación eléctrica.

\section{PROBLEMA}

En el sector industrial y de servicios, las empresas, especialmente las medianas y pequeñas (mypes), presentan sobrecostos de energía eléctrica debido, en parte, a la deficiente gestión de cargas eléctricas (load management) y al alto costo de sistemas de control convencionales. Esta situación se ve reflejada en la presencia de demandas de potencia eléctrica no deseadas, las cuales se producen cuando la potencia eléctrica utilizada por el cliente supera un límite fijado en el acuerdo tarifario (demanda máxima contratada), elevando los montos de facturación mensual por servicio de electricidad (síntomas).

Para llevar a cabo esta investigación se revisó inicialmente la literatura especializada en uso eficiente de energía eléctrica, como los trabajos de Peter Radgen y Klaus Brinkmann; asimismo, de acuerdo con la metodología de investigación empleada, se fijaron los límites de esta en cuanto a tema, lugar y tiempo, mediante la pregunta ¿qué herramienta de gestión de demanda eléctrica se deben implementar en empresas industriales y de servicios del país, especialmente mypes, para mejorar la gestión de energía eléctrica, al reducir sobrecostos de facturación y, de esta forma, hacerse más competitivas? 
Figura 1

Demanda de potencia

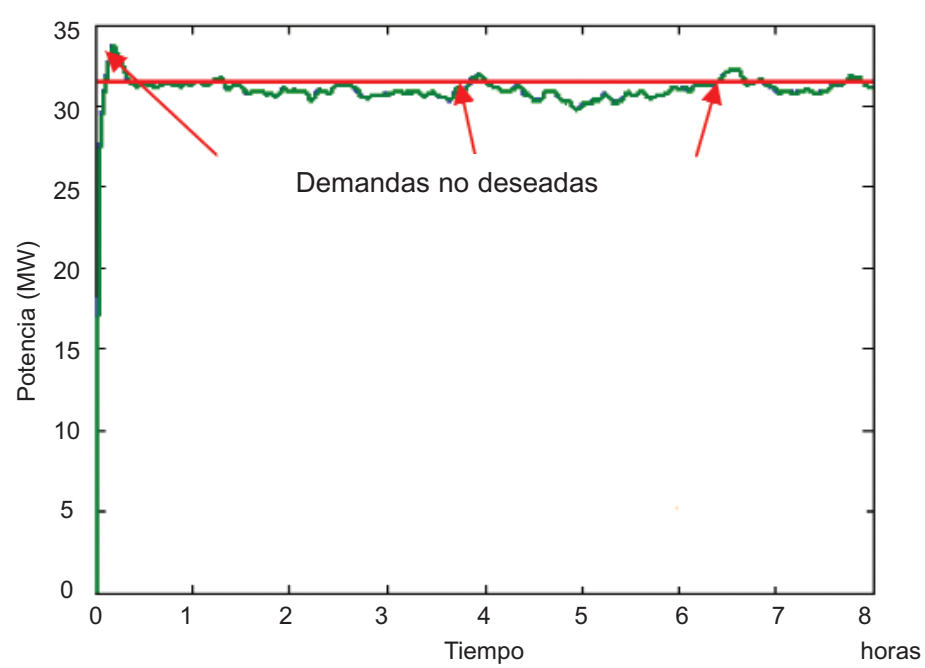

Fuente: Sección de molienda de la concentradora Toquepala de Southern Perú.

\section{OBJETIVOS}

\subsection{Objetivo general}

El objetivo de la propuesta es mejorar el uso de energía eléctrica en las empresas industriales y de servicios, especialmente en las mypes, al reducir los sobrecostos producidos por la demanda no deseada (picos de demanda de potencia eléctrica) mediante un controlador digital de demanda máxima, de alta precisión y bajo costo, que monitoree dicha potencia eléctrica y evite que se supere el umbral de demanda máxima contratada, desactivando por intervalos de tiempo ciertos equipos que no afectan la continuidad del negocio (iluminación, ventiladores, etcétera).

\subsection{Objetivos específicos}

Los objetivos específicos fueron los siguientes:

- Mejorar la gestión de demanda de potencia eléctrica.

- Desarrollar un dispositivo electrónico de alta precisión, bajo costo y tecnología propia para eliminar las demandas no deseadas.

- Reducir sobrecostos de facturación de energía eléctrica. 


\subsection{Finalidad}

Promover una cultura de uso eficiente de energía eléctrica, a partir de metodologías adecuadas y productos tecnológicos innovadores, que mejoren la competitividad de las empresas industriales nacionales y permitan la generación de divisas para el desarrollo del país.

\section{METODOLOGÍA}

Se ha utilizado el método deductivo, tomando en cuenta trabajos anteriores sobre equipos de control $[6,7]$ y sistemas inteligentes de pronóstico de series temporales [8-14], que ha permitido conocer el estado de la técnica en ambos casos.

Se eligió la sección de molienda de la concentradora Toquepala de Southern Peru como unidad de observación, y su sistema eléctrico como unidad de análisis para llevar a cabo esta investigación, pues esta instalación presenta demandas no deseadas que encarecen los costos de su proceso productivo. Esta planta (la sección de molienda) posee características muy similares a las que podría tener una mype; es decir, equipos principales que no pueden ser manipulados por el controlador de demanda y los complementarios pero que sí se adecúan a esta lógica de control. Además, se tenía la facilidad de poder implementar la lógica de control propuesta en esta planta, y se contaba con data confiable para su simulación.

Inicialmente, se recopiló información de campo para tener datos necesarios que sirvieran de base para el diseño del controlador digital, en especial para el módulo predictivo de demanda eléctrica, y para simular la sección de molienda de la concentradora en la cual se pudiera validar la eficacia del dispositivo electrónico. Esta planta virtual se desarrolló inicialmente con el software Matlab para evaluar la confiabilidad del modelo y posteriormente se trasladó al software Labview para hacer las pruebas con el hardware desarrollado. Esta validación del controlador de demanda se llevó a cabo inicialmente en el laboratorio de Mecatrónica (LAB-MT) de la Facultad de Ingeniería Mecánica de la Universidad Nacional de Ingeniería durante la primera semana de abril del 2010 y posteriormente, a fines del mismo mes, en la Facultad de Ingeniería Electrónica de la Universidad Privada de Tacna. 


\section{HIPÓTESIS DE LA INVESTIGACIÓN}

Para la hipótesis, se definieron las siguientes variables de investigación:

- Variables dependientes: gestión de demanda eléctrica, sobrecostos de energía eléctrica.

- Variables independientes: controlador digital predictivo de demanda, estructura tarifaria, modelo predictivo y modelo de control convencional.

De esta forma, la hipótesis establece que si se diseña y construye un controlador digital predictivo de demanda máxima de potencia eléctrica, que comprenda un arreglo de equipos de acuerdo con las tarifas e incluya un modelo predictivo y un modelo de control convencional, se mejorará la gestión de demanda de potencia eléctrica y se reducirán los sobrecostos de energía eléctrica, producidos por la demanda no deseada (picos de demanda de potencia eléctrica).

\section{POBLACIÓN Y MUESTRA}

Para las pruebas de campo del controlador digital de demanda de potencia eléctrica se ha considerado una población infinita de lecturas de potencia eléctrica en períodos de 15 minutos de la sección de molienda de la concentradora Toquepala. En ese sentido, para determinar el tamaño de muestra de lecturas de potencia eléctrica representativa, que es de $42,68 \approx 43$, se empleó la fórmula de tamaño de muestra para proporciones, según la siguiente expresión:

Donde:

$$
n=\frac{Z_{i}^{2} p(1-p)}{d^{2}}
$$

$Z_{i}$ : Nivel de confianza de 95\% (en una distribución normal el valor en tablas que corresponde es 1,96$)$.

$p=$ proporción esperada (en este caso $50 \%=0,5$ ).

$q=1-\mathrm{p}$ (en este caso 0,5$)$.

$\mathrm{d}=$ precisión de $15 \%$ (en este caso el valor es 0,0225 ). 


\section{ESTRUCTURA DESGLOSABLE DEL TRABAJO}

El proyecto se dividió en siete resultados (productos o resultados medibles de un proyecto final):

E1. Diseño de matriz $\mathbf{X}$ para operación de equipos.

E2. Código de programación para el controlador.

E3. Circuito eléctrico del controlador.

E4. Módulo predictivo.

E5. Simulación de planta de molienda de la concentradora Toquepala.

E6. Simulación del controlador en la concentradora.

E7. Implementación y validación del hardware.

Asimismo, estos resultados se han dividido en subresultados (paquetes de trabajo y actividades) para facilitar la gestión del proyecto, según el detalle mostrado en el cuadro 1.

\section{Cuadro 1 \\ Resultados del proyecto}

\begin{tabular}{|c|c|c|c|c|}
\hline \multicolumn{5}{|c|}{ Controlador digital de demanda de energía eléctrica } \\
\hline \multicolumn{2}{|r|}{ Resultados } & Actividades & Indicadores & Paquetes de trabajo \\
\hline \multirow{5}{*}{ E1 } & \multirow{5}{*}{$\begin{array}{l}\text { Diseño de la } \\
\text { matriz X para la } \\
\text { operación de los } \\
\text { equipos }\end{array}$} & \multirow{2}{*}{$\begin{array}{l}\text { Diseñar la matriz } X \\
\text { que genere una de- } \\
\text { manda lo más cerca- } \\
\text { na y no mayor a MW }\end{array}$} & \multirow{2}{*}{$\begin{array}{l}\text { Demanda total de equi- } \\
\text { pos por cada hora según } \\
\text { la matriz X }\end{array}$} & $\begin{array}{l}\text { Hallar la potencia nominal } \\
\text { de cada equipo }\end{array}$ \\
\hline & & & & $\begin{array}{l}\text { Maximizar la función de } \\
\text { potencia }\end{array}$ \\
\hline & & \multirow{3}{*}{$\begin{array}{l}\text { Diseñar la matriz } X \\
\text { que brinde un costo } \\
\text { de energía lo más cer- } \\
\text { cano y no mayor a } \$\end{array}$} & $\begin{array}{l}\text { Consumo total de ener- } \\
\text { gía (equipos) por cada } \\
\text { hora según la matriz } X\end{array}$ & $\begin{array}{l}\text { Determinar el consumo de } \\
\text { energía por hora }\end{array}$ \\
\hline & & & \multirow{2}{*}{$\begin{array}{l}\text { Costo de energía por } \\
\text { cada hora según la } \\
\text { matriz } X\end{array}$} & $\begin{array}{l}\text { Determinar el costo de } \\
\text { energía por hora }\end{array}$ \\
\hline & & & & $\begin{array}{l}\text { Maximizar la función de } \\
\text { costo de energía por hora }\end{array}$ \\
\hline
\end{tabular}


(continuación)

\begin{tabular}{|c|c|c|c|c|}
\hline \multicolumn{5}{|c|}{ Controlador digital de demanda de energía eléctrica } \\
\hline \multicolumn{2}{|r|}{ Resultados } & Actividades & Indicadores & Paquetes de trabajo \\
\hline \multirow{7}{*}{ E2 } & \multirow{7}{*}{$\begin{array}{l}\text { Código de pro- } \\
\text { gramación en el } \\
\text { software Code } \\
\text { Composser para } \\
\text { el controlador }\end{array}$} & \multirow{3}{*}{$\begin{array}{l}\text { Desarrollar el código } \\
\text { de la ley de control } \\
\text { con el tiempo de eje- } \\
\text { cución menor a } 1 \\
\text { minuto }\end{array}$} & $\begin{array}{l}\text { Porcentaje de desarrollo } \\
\text { del código de la ley del } \\
\text { control }\end{array}$ & \\
\hline & & & \multirow{2}{*}{$\begin{array}{l}\text { Tiempo de ejecución del } \\
\text { código de la ley de } \\
\text { control }\end{array}$} & $\begin{array}{l}\text { Configurar los tempori- } \\
\text { zadores }\end{array}$ \\
\hline & & & & $\begin{array}{l}\text { Hallar la velocidad de eje- } \\
\text { cución de instrucciones }\end{array}$ \\
\hline & & $\begin{array}{l}\text { Implementar un reloj } \\
\text { con un retardo de } 0 \\
\text { segundos }\end{array}$ & Retardo por día del reloj & $\begin{array}{l}\text { Comparar el tiempo dado } \\
\text { por el reloj con el tiempo } \\
\text { dado }\end{array}$ \\
\hline & & \multirow{3}{*}{$\begin{array}{l}\text { Desarrollar el código } \\
\text { de la red neuronal del } \\
\text { módulo de predicción } \\
\text { con un tiempo de eje- } \\
\text { cución menor a } 1 \\
\text { minuto }\end{array}$} & $\begin{array}{l}\text { Porcentaje de desarrollo } \\
\text { del código de la red neu- } \\
\text { ronal }\end{array}$ & $\begin{array}{l}\text { Hacer el código de la red } \\
\text { neuronal }\end{array}$ \\
\hline & & & \multirow{2}{*}{$\begin{array}{l}\text { Tiempo de ejecución del } \\
\text { código de la red neuronal }\end{array}$} & $\begin{array}{l}\text { Configurar los tempori- } \\
\text { zadores }\end{array}$ \\
\hline & & & & $\begin{array}{l}\text { Hallar la velocidad de eje- } \\
\text { cución de instrucciones }\end{array}$ \\
\hline \multirow{3}{*}{ E3 } & \multirow{3}{*}{$\begin{array}{l}\text { Diseñar la placa } \\
\text { del circuito del } \\
\text { controlador }\end{array}$} & \multirow{3}{*}{$\begin{array}{l}\text { Diseñar la placa } \\
\text { del circuito del contro- } \\
\text { lador }\end{array}$} & \multirow{3}{*}{$\begin{array}{l}\text { Diseñar la placa del cir- } \\
\text { cuito del controlador }\end{array}$} & $\begin{array}{l}\text { Realizar el esquema del } \\
\text { controlador }\end{array}$ \\
\hline & & & & $\begin{array}{l}\text { Diseñar la placa del circui- } \\
\text { to del controlador }\end{array}$ \\
\hline & & & & $\begin{array}{l}\text { Diseñar la placa del circui- } \\
\text { to del controlador }\end{array}$ \\
\hline
\end{tabular}


(continuación)

\begin{tabular}{|c|c|c|c|c|}
\hline \multicolumn{5}{|c|}{ Controlador digital de demanda de energía eléctrica } \\
\hline \multicolumn{2}{|r|}{ Resultados } & Actividades & Indicadores & Paquetes de trabajo \\
\hline \multirow{3}{*}{ E4 } & \multirow{3}{*}{ Modelo predictivo } & Construir red neuronal & Porcentaje de ejecución & Tomar los datos \\
\hline & & $\begin{array}{l}\text { Definir algoritmo, fun- } \\
\text { ciones y patrones }\end{array}$ & Precisión alcanzada & $\begin{array}{l}\text { Desarrollar el módulo de } \\
\text { predicción }\end{array}$ \\
\hline & & $\begin{array}{l}\text { Validar pronóstico de } \\
\text { demanda }\end{array}$ & $\begin{array}{l}\text { Error cuadrático medio del } \\
\text { pronóstico de demanda }\end{array}$ & Validar el módulo \\
\hline \multirow{3}{*}{ E5 } & \multirow{3}{*}{$\begin{array}{l}\text { Simulación de } \\
\text { la sección de la } \\
\text { molienda de la } \\
\text { concentradora } \\
\text { Toquepala }\end{array}$} & \multirow{3}{*}{$\begin{array}{l}\text { Tener una base de } \\
\text { referencia para las } \\
\text { pruebas de validación }\end{array}$} & \multirow{3}{*}{$\begin{array}{l}\text { Error cuadrático medio de } \\
\text { las principales variables }\end{array}$} & Entender el proceso \\
\hline & & & & Desarrollar la simulación \\
\hline & & & & Validar la simulación \\
\hline E6 & $\begin{array}{l}\text { Simulación del } \\
\text { controlador en } \\
\text { la sección } \\
\text { molienda }\end{array}$ & $\begin{array}{l}\text { Modelar y simular la } \\
\text { planta de la molienda }\end{array}$ & $\begin{array}{l}\text { Error cuadrático medio de } \\
\text { demanda de potencia, } \\
\text { energía y variables de } \\
\text { producción }\end{array}$ & $\begin{array}{l}\text { Desarrollar y validar la } \\
\text { simulación }\end{array}$ \\
\hline \multirow{5}{*}{ E7 } & \multirow{5}{*}{$\begin{array}{l}\text { Controlador } \\
\text { validado } \\
\text { mediante prue- } \\
\text { bas en la planta } \\
\text { simulada de la } \\
\text { sección molien- } \\
\text { da de la con- } \\
\text { centradora } \\
\text { Toquepala }\end{array}$} & $\begin{array}{l}\text { Muestrear la deman- } \\
\text { da de la concentrado- } \\
\text { ra con un período de } \\
\text { muestreo de } 15 \\
\text { minutos } \\
\end{array}$ & Período de muestreo & $\begin{array}{l}\text { Sintonizar el reloj con el } \\
\text { período de muestreo }\end{array}$ \\
\hline & & \multirow{4}{*}{$\begin{array}{l}\text { Ejecutar el controla- } \\
\text { dor obteniendo un } \\
\text { porcentaje de deman- } \\
\text { da por encima del } \\
\text { umbral } 0 \%\end{array}$} & Número de equipos & Sintonizar la matriz $\mathrm{X}$ \\
\hline & & & $\begin{array}{l}\text { Demanda actual de la } \\
\text { concentradora }\end{array}$ & $\begin{array}{l}\text { Configurar la operación } \\
\text { del conversor } A D \text { con el } \\
\text { controlador }\end{array}$ \\
\hline & & & $\begin{array}{l}\text { Error en predecir la } \\
\text { demanda de la concen- } \\
\text { tradora }\end{array}$ & $\begin{array}{l}\text { Entrenar la red neuronal } \\
\text { con valor actual de la } \\
\text { concentradora }\end{array}$ \\
\hline & & & $\begin{array}{l}\text { Demanda total que supe- } \\
\text { ra el límite }\end{array}$ & $\begin{array}{l}\text { Sintonizar las variables } \\
\text { de la concentradora }\end{array}$ \\
\hline
\end{tabular}

Elaboración propia. 


\section{DESARROLLO Y CONSTRUCCIÓN DEL EOUIPO}

En este capítulo se detallan los resultados del proyecto desde la etapa de desarrollo del controlador, pasando por su construcción misma hasta su implementación y validación en la planta virtual de la sección molienda.

\subsection{Diseño de la matriz X para operación de equipos}

Para encontrar el conjunto óptimo de los grupos de equipos que deben operar en una planta en cada período de tiempo, generalmente una hora, se ha desarrollado un modelo de programación lineal binaria. La variable principal de este modelo es $x_{i j}$ definida como:

$x_{i j}=1$, si el grupo de equipos $i$ funciona en el horario $j$, con $i=1, \ldots$, m y $j=1, \ldots$, n.

$x_{i j}=0$, si el grupo de equipos $i$ no funciona en el horario $j$, con $i=$ $1, \ldots, \mathrm{m} \mathrm{y} j=1, \ldots, \mathrm{n}$.

$c_{i j}$ : costo de operación del grupo de equipos $i$ funcionando en el horario $j$.

$q_{i \text { : }}$ cargas asociadas a los grupos de equipos i.

El modelo encuentra el conjunto óptimo de equipos que deben operar en los horarios prefijados para minimizar el costo de facturación.

La función objetivo se expresa como Min Z; es decir, la sumatoria de términos formados por los costos $\mathrm{c}_{\mathrm{ij}}$, y sus grupos asociados de equipos $x_{i j}$, según la siguiente expresión matemática:

$$
\operatorname{Min} \mathrm{Z}=\sum_{i=1}^{m} \sum_{j=1}^{n} c_{i j} x_{i j}
$$

Sujeto a las siguientes restricciones de operación:

$$
\begin{aligned}
& \sum_{i=1}^{m} x_{i j} \geq 1, \mathrm{j}=1, \ldots, \mathrm{n} \\
& \sum_{j=1}^{n} x_{i j} \geq 1, \mathrm{i}=1, \ldots, \mathrm{m}
\end{aligned}
$$


La restricción de demanda máxima contratada:

$$
\begin{aligned}
& \sum_{i=1}^{m} \sum_{j=1}^{n} q_{i} x_{i j} \leq d_{\max } \\
& x_{i j} \in\{0,1\}
\end{aligned}
$$

\subsection{Código de programación para el controlador}

En este resultado se desarrolló el código del controlador en lenguaje C. Este código contempla todas las instrucciones que incluyen el módulo de predicción con la red neuronal del tipo Nonlinear AutoRegressive with eXogenous input - NARX, que es un modelo de red que establece una relación entre data pasada de entrada-salida y salidas predichas, además del control Proporcional Integral Derivativo - PID para los arreglos de la matriz $\mathbf{X}$ (véase la figura 2). Asimismo, se determinaron las instrucciones para una correcta comunicación con los periféricos, que son el convertidor analógico-digital y la pantalla de cristal líquido. Además, se consideró un periodo de muestreo de 15 minutos y un tiempo de ejecución de cada ciclo de operación del controlador menor a 1 minuto para asegurar su correcta operación.

\subsection{Circuito eléctrico del controlador}

El diagrama de bloques del sistema de control de demanda se muestra en la figura 3 , en la cual se observa que el controlador propiamente dicho es el PIC18F4550 que contiene la ley de control, el módulo de pronóstico de demanda y la matriz $\mathbf{X}$; también se aprecian los periféricos, como el convertidor analógico-digital con su filtro, la pantalla de cristal líquido y el circuito de protección para las salidas digitales.

Por otro lado, el bloque set-point (valor deseado o punto de ajuste de alguna variable en un sistema de control automático) corresponde al valor de la demanda contratada o demanda máxima, que es el setpoint del sistema de control. 
Controlador digital predictivo de demanda máxima de potencia eléctrica

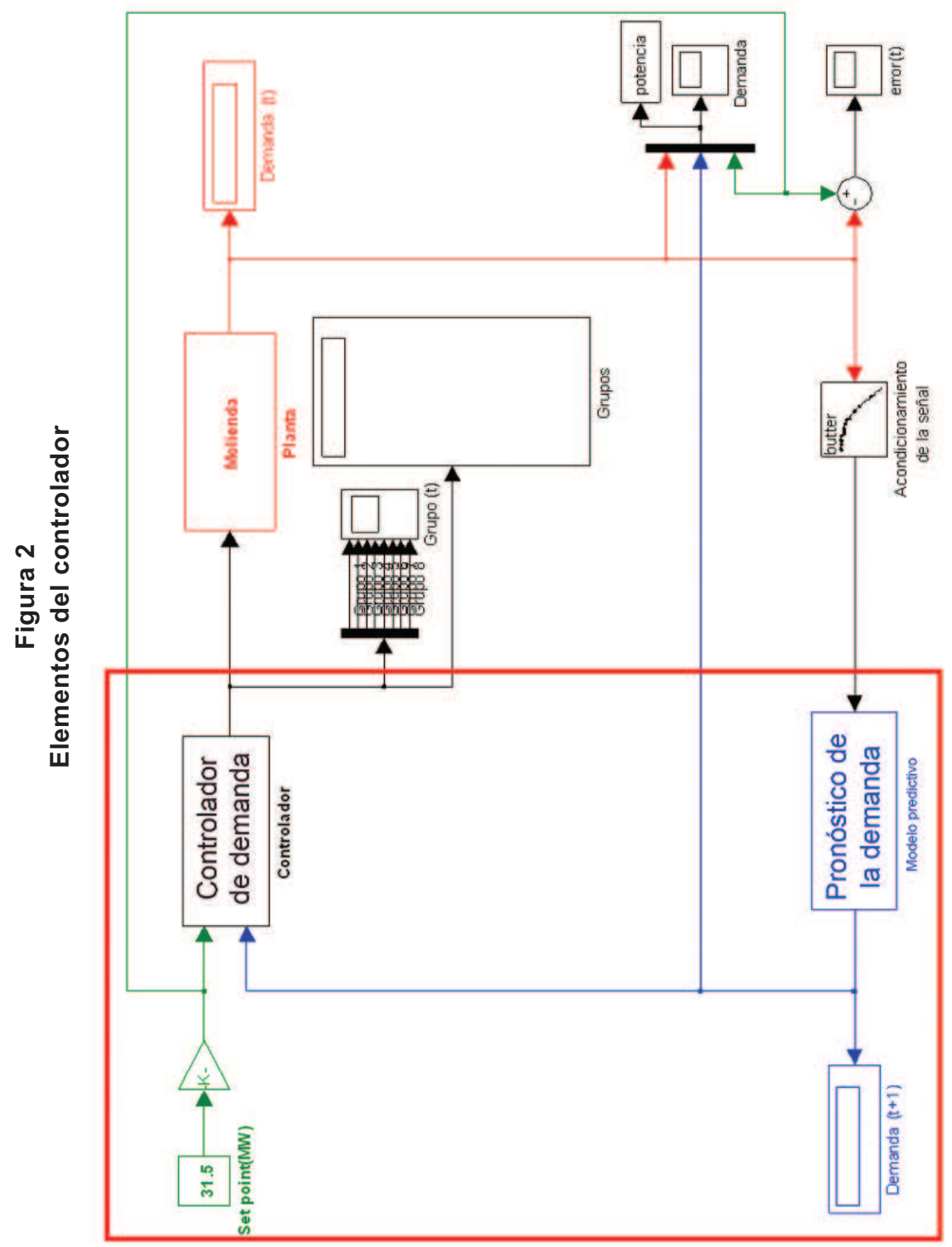

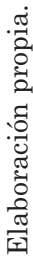


Figura 3

Diagrama de bloques del dispositivo electrónico

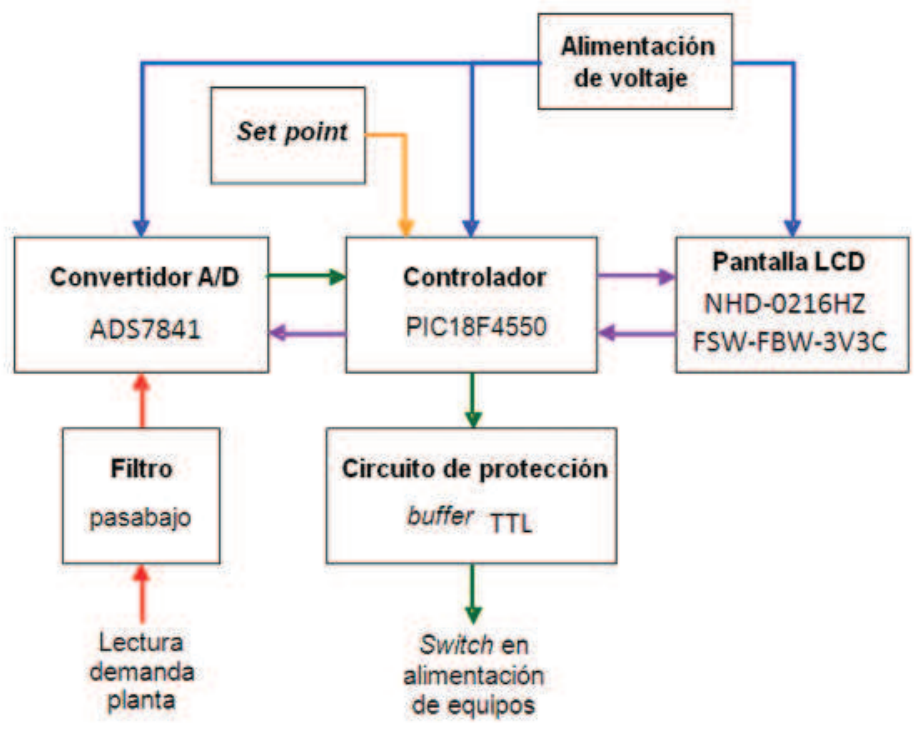

Elaboración propia.

En la implementación, el controlador ha sido instalado en la tarjeta de desarrollo; mientras que el convertidor analógico-digital, el filtro pasabajo, la pantalla de cristal líquido y el circuito de protección están en una tarjeta electrónica adicional cuyo diseño se explica a continuación.

Primero, se ha empleado un condensador en la entrada analógica que funciona como un filtro pasabajo para reducir el nivel de ruido en la señal. Luego, el convertidor analógico-digital está implementado mediante el circuito integrado ADS7841. Este convertidor puede digitalizar cuatro señales analógicas en forma secuencial y transmitir el valor en forma serial con una resolución de 12 bits.

Para proteger en cierto grado y aislar el PIC18F4550 de los circuitos electrónicos de mayor potencia que está instalado a la entrada de los grupos de equipos se empleó un buffer con colector abierto.

Finalmente, para observar los valores de la demanda real pronosticada se usó la pantalla de cristal líquido NHD-0216HZ-FSW-FBW- 
3V3C. Esta pantalla cuenta con $2 \times 16$ caracteres con una resolución de $5 \times 7$ cada uno.

\subsection{Modelo predictivo}

Está conformado por una red neuronal NARX, que predice la demanda de la planta basada en la tendencia que esta presenta con un error menor de 3\% alrededor del valor real, según las investigaciones de Meisenbach. Este modelo utiliza las demandas históricas para entrenar la red, y considera los patrones calendario de producción y la demanda anterior, que se leen cada 15 minutos. Este valor pronosticado de la demanda de energía eléctrica del siguiente período, también de 15 minutos, es comparado con el set point de esta misma variable.

En la figura 4 se presenta la arquitectura de red del modelo predictivo basada en una estructura neuronal dinámica NARX modificada. Esta red neuronal es una red recurrente con dos retardos en la salida y una capa oculta. Tanto la capa oculta como la de salida tienen por función de transferencia la función tansig, que es una función de transferencia no lineal usada para entrenar redes neuronales también llamada función tangente sigmoidal hiperbólica.

Figura 4

Arquitectura de red del modelo predictivo

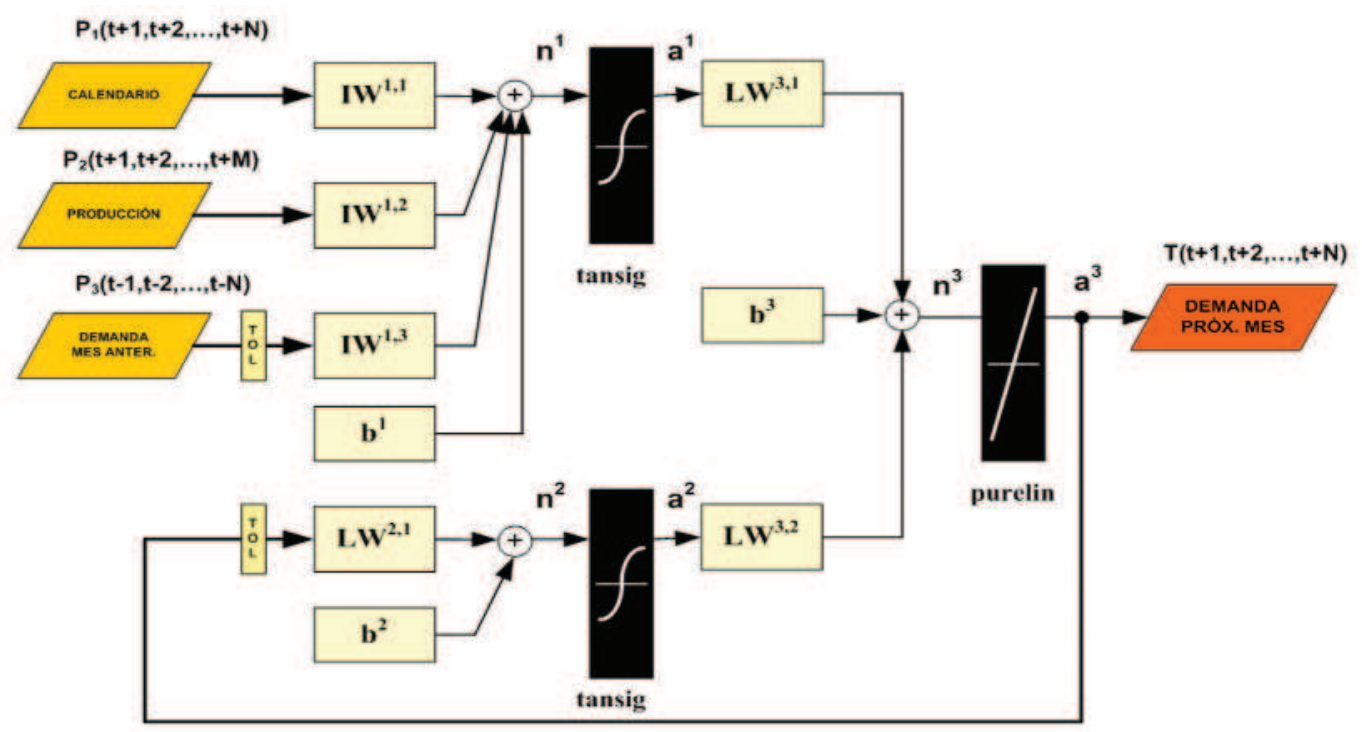

Elaboración propia. 
a) Patrones de entrada.- Los factores que influyen directamente en la demanda eléctrica son: calendario, producción, demanda anterior y temperatura. Sin embargo, debido a la dificultad de contar con valores exactos de la temperatura, solo se consideraron los tres primeros patrones de entrada para la red neuronal del modelo de predicción. Un ejemplo de los datos obtenidos en su forma original y en su nueva escala $[0,1]$ se presenta en el cuadro 2 :

\section{Cuadro 2}

Tratamiento de los datos de entrada

\begin{tabular}{|c|c|c|c|c|c|c|c|c|}
\hline \multicolumn{9}{|c|}{ Información de entrada en su forma original } \\
\hline $1 / 4$ hora & hora & día & sem. & mes & Prod1(TM/h) & Prod2(TM/h) & Prod3(TM/h) & Demanda(t-1) \\
\hline 1 & 0 & 2 & 3 & 4 & 27099 & 1012.15 & 33252 & 30 \\
\hline \multicolumn{9}{|c|}{ Información de entrada en nueva escala } \\
\hline 0.33 & 0 & 0.33 & 0.75 & 0.36 & 0.677 & 0.86309 & 0.738 & 1 \\
\hline
\end{tabular}

Elaboración propia.

Para la normalización, las variables consideradas en el modelo han sido linealmente escaladas (linear scaling) entre 0 y 1 . Este procedimiento consiste simplemente en modificar la escala de las variables atendiendo a su valor mínimo y máximo para obtener series más homogéneas.

b) Salidas o targets.- De acuerdo con el objetivo del modelo predictivo, las salidas fueron:

- Demanda del mes próximo. Es un vector de $1 \times \mathrm{N}$, donde $\mathrm{N}$ es el valor de demanda pronosticada de un mes futuro; es decir, en diciembre de un año se puede calcular la demanda de energía eléctrica de enero a diciembre del año siguiente.

- Consumo del mes próximo. Es un valor (1 x 1) obtenido directamente de la data de demanda pronosticada, considerando el algoritmo base de la facturación mensual.

c) Prueba del modelo predictivo.- Con la red entrenada y el porcentaje de error de $0,51 \%$ (para entrenamiento), se procedió a seleccionar un mes del menú desplegable para su predicción y obtención de 
variables. Los datos formales para la validación se obtuvieron del servidor IION, que contiene los reportes del Departamento de Sistemas de Potencia de Southern Perú.

Para la validación del módulo se utilizó el error cuadrático medio. El error cuadrático medio en porcentaje se define como:

$$
\% e_{r m s}=\frac{\sum_{i=1}^{N}\left(\frac{p_{i}-r_{i}}{r_{i}}\right)^{2}}{N} \times 100
$$

Donde:

$p_{i}:$ Dato simulado

$r_{i}$ : Dato real

$N$ : Número de muestras

El cuadro 3 muestra el error cuadrático medio del modelo predictivo, validado con los datos de facturación de octubre del 2008; es decir: $0,4862 \%$.

\begin{tabular}{cc}
\multicolumn{2}{c}{ Cuadro 3} \\
Error cuadrático medio \\
\hline Mes & ${ }^{\%_{\text {erms }}}$ \\
\hline Oct-08 & $0,4862 \%$ \\
\hline
\end{tabular}

Fuente: Sistema de potencia de Southern Perú.

Elaboración propia.

\subsection{Simulación de planta de molienda}

Para probar el controlador de demanda se modeló y simuló la sección de molienda de la concentradora Toquepala. Este módulo ha sido construido en Simulink y simulado con el método "Dormand-Prince" para estados continuos. La idea básica para su construcción fue la separación del módulo por secciones, tal como se presenta en la figura 5 . 
Figura 5

Diagrama del módulo de simulación de la planta de molienda

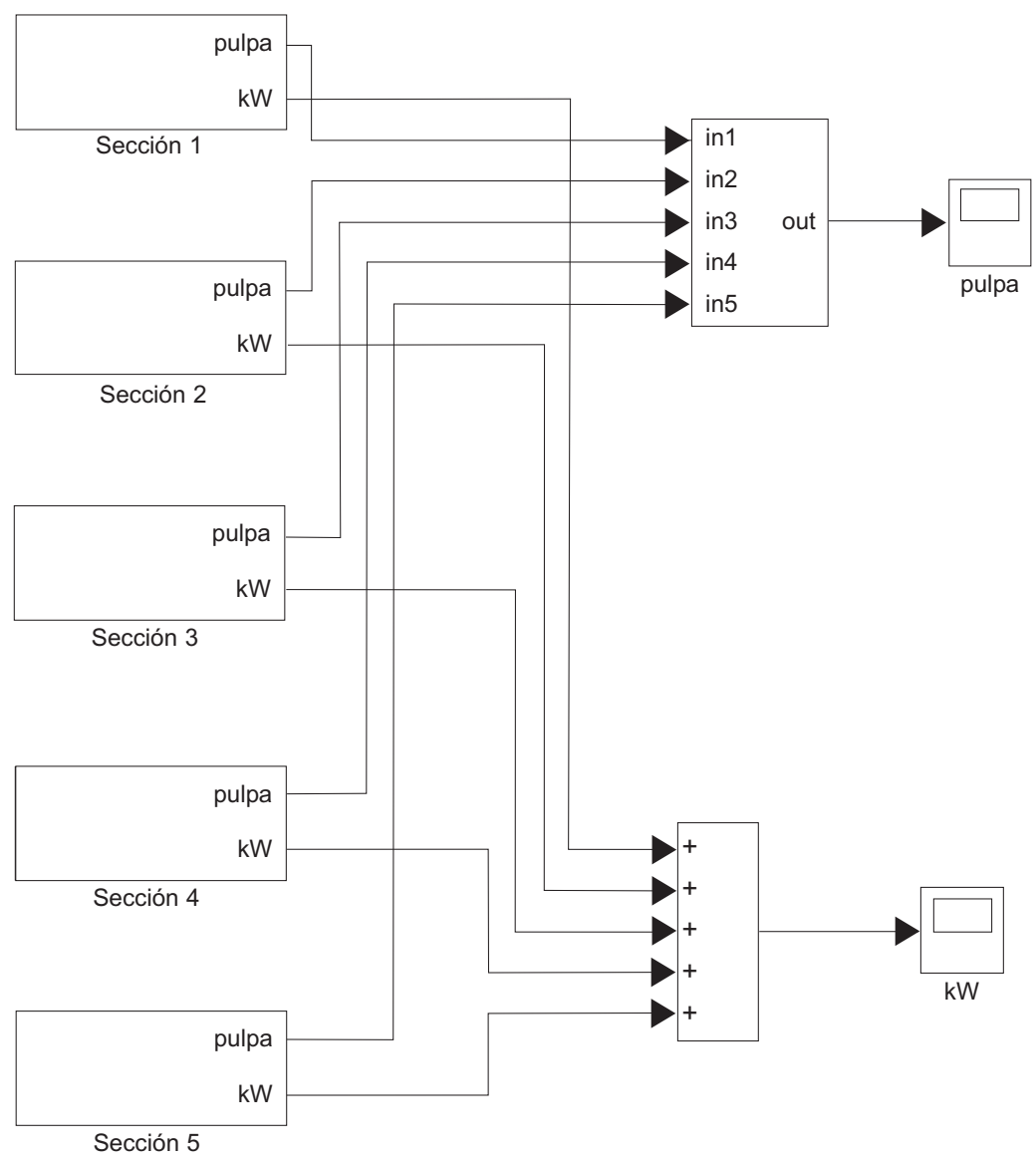

Fuente: Concentradora Toquepala.

El módulo de simulación de la etapa de molienda de la concentradora Toquepala se desarrolló al 100\%, comprobándose durante la validación del modelo que este reproduce la potencia eléctrica y la energía con un error cuadrático medio total de $2,33 \%$ y de $0,39 \%$, respectivamente. Para esta evaluación se compararon las lecturas de potencias activas simuladas versus las lecturas del System PI (Process Information), el cual permite obtener información avanzada de datos de procesos de una planta de la concentradora Toquepala. 


\subsection{Simulación del controlador en la sección de molienda}

De acuerdo con la metodología propuesta, se ha simulado el modelo de control de demanda para evaluar su acción en un sistema eléctrico, según se aprecia en la figura 6. En la planta se consideraron 8 grupos de equipos, que han sido modelados mediante un número aleatorio con distribución gaussiana y media igual al valor esperado de su demanda; y han sido filtrados para simular un proceso continuo.

\section{Figura 6}

\section{Pantalla del módulo de simulación del controlador}

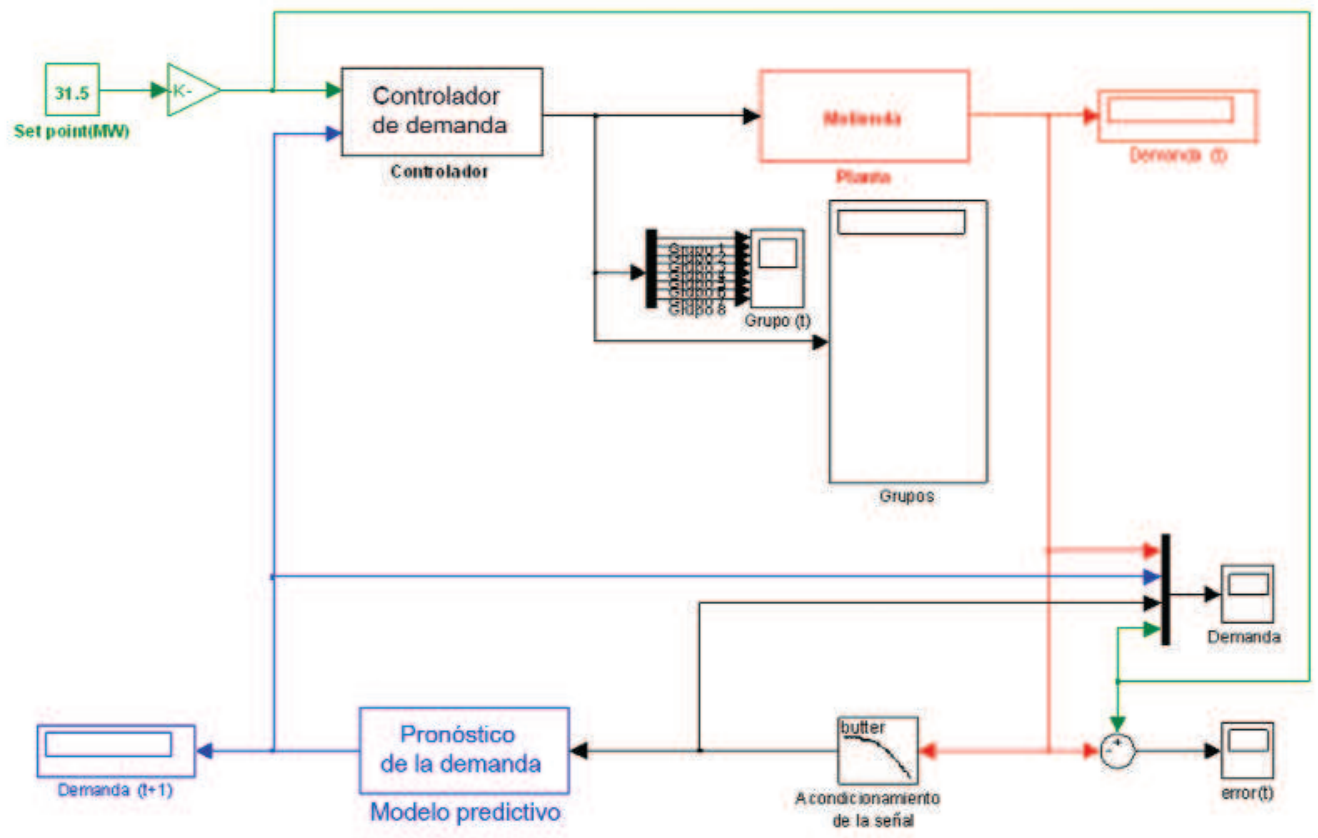

Elaboración propia.

Las variables de entrada consideradas son el set point de la demanda de energía eléctrica igual a 31,6 MW y el valor pronosticado de dicha demanda $\mathrm{d}(t+1)$, calculado por el modelo predictivo para los 15 minutos siguientes al período $d(t)$. Esta variable de salida realimenta la red NARX del módulo predictivo. 
El modelo predictivo está conformado por una red neuronal NARX, que predice la demanda de la planta sobre la base de la tendencia que esta presenta. El siguiente bloque es el controlador PID, que corrige la demanda activando o desactivando a cada grupo; sin embargo, para cerrar el lazo se transformó el valor del controlador que es continuo respecto al de la planta que es binario.

Se creó así el último bloque -último en entrar, primero en salir (LIFO, por sus siglas en inglés) — que crea la disciplina de operación de cada equipo por horas. El grupo LIFO tiene por entradas al tiempo y la señal del controlador. El tiempo está dado en horas en un rango entero de 1 a 24. La señal del controlador permite seleccionar en el calendario (bloque con matriz $x_{i j}$ ) qué equipo puede entrar en funcionamiento en el periodo siguiente para no superar el umbral de demanda deseado.

La matriz $X$ es obtenida mediante el modelo de programación lineal, que representa el conjunto óptimo formado por las variables $x_{i j}$ asociadas al grupo de equipos $i$ en el horario $j$, y se calcula después de haberlos agrupado adecuadamente, sobre la base de un censo real en cada planta que permita determinar las cargas de cada grupo, así como conociendo la tarifa horaria $j$ de cada hora y el presupuesto mensual.

Luego se multiplica por la demanda característica (valor medio o máximo) de cada grupo y se determina la demanda acumulada (demanda solo con equipo 1 , demanda con equipo 1 y 2 , demanda con 1,2 y 3 , etcétera). La demanda acumulada se compara con la señal de control, seleccionando aquella cuya demanda es inmediatamente menor a la señal de control. Esta comparación permite identificar los equipos que deben seguir activados.

El error es calculado entre el set-point de 31,6 MW y el valor pronosticado por el modelo predictivo, que se ingresa al controlador para establecer cuál debería ser la demanda. Luego, el bloque LIFO transforma este valor en un vector que indica qué grupos deben seguir encendidos.

En la simulación se pudo encontrar el siguiente arreglo de grupos de equipos: $100,35,25,10,5,10,5,5$.

El modelo de control mantiene la demanda por debajo de los 31,6 MW. Cuando la demanda pronosticada es menor a 31,6 MW el controlador deja de funcionar, permitiendo a todos los grupos entrar en operación. Por el contrario, cuando el controlador pronostica una demanda no deseada, se anticipa a dicho evento y desactiva los grupos 
de cargas para no superar el umbral de demanda máxima (línea celeste), logrando mantener la demanda de potencia (línea roja) siempre por debajo de aquella.

Figura 7

\section{Curva de demanda}

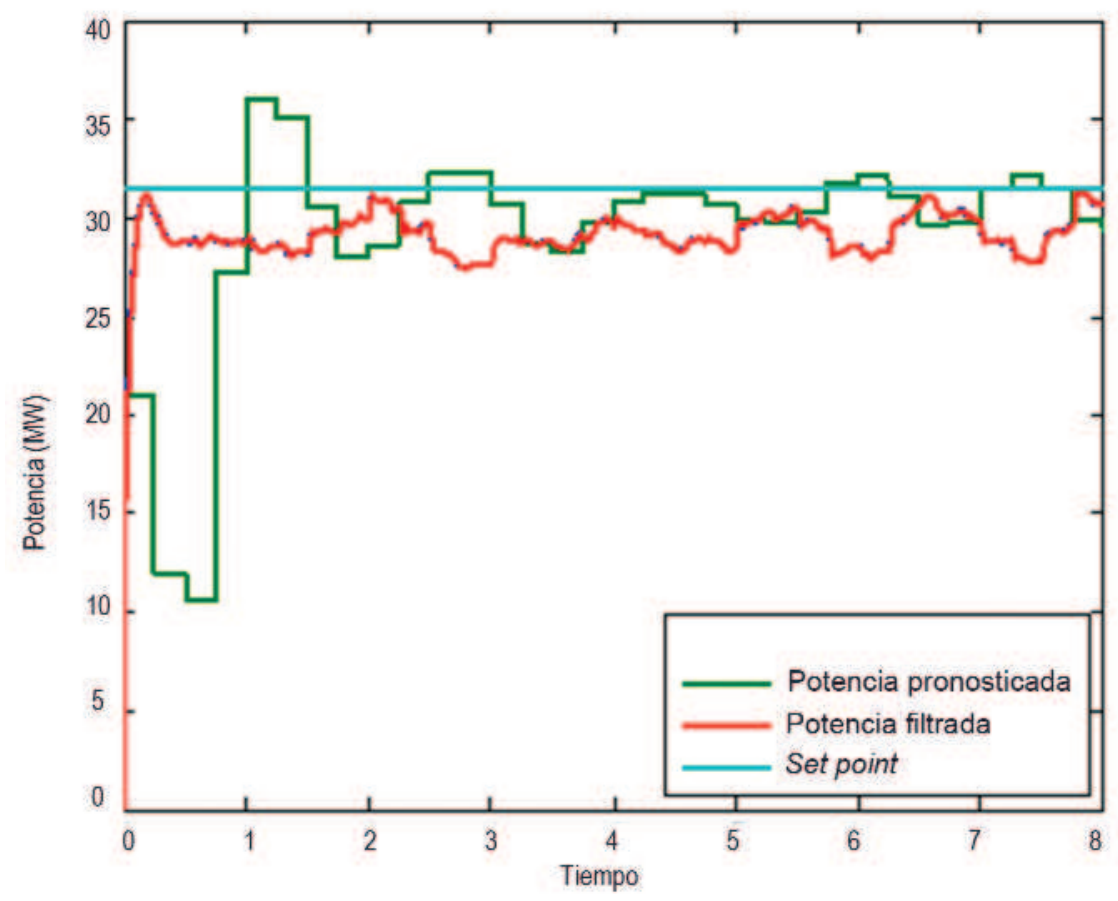

Elaboración propia.

Línea celeste: set point o valor deseado de demanda

Línea verde: demanda pronosticada

Línea roja: demanda con controlador

\subsection{Validación del controlador en hardware}

Las variables primarias de interés del proceso fueron el tiempo, el grupo de señales de voltaje de entrada de los grupos de operación y el voltaje de demanda de potencia, puesto que estas variables muestran el funcionamiento del lazo de control y pueden ser adquiridas directamente. 
Las variables secundarias fueron el voltaje pronosticado de demanda, la señal de control, el error de control, entre otras, las cuales son parte secundaria del proceso y no pueden ser adquiridas directamente.

Habiéndose establecido el tamaño de la muestra de 43 lecturas de 15 minutos, se consideró una corrida cercana de 40 segundos, que representa un tamaño de muestra de 40 lecturas (tiempo de trabajo de 600 minutos) con tiempo de muestreo de 15 minutos; es decir, que 1 segundo en la simulación representa 15 minutos en el proceso real.

$$
\begin{aligned}
& t_{\text {real }}=\{0,1, \ldots, 39,40\} \text { segundos } \\
& t_{\text {representado }}=\{0,15, \ldots, 585,600\} \text { minutos. }
\end{aligned}
$$

Por último, las asunciones de operación tomadas en cuenta para la validación del controlador de potencia fueron las siguientes:

- Operación normal de la planta de molienda.

- Plan de producción de la concentradora.

- Alimentación de energía eléctrica constante.

Con el algoritmo del controlador corregido y validado se procedió a grabar este programa en el PIC18F4550, para luego validar su funcionamiento con la planta simulada en LabView (véase la figura 8), mediante pruebas de laboratorio que se hicieron con el uso de un dispositivo de adquisición de datos (DAQ) modelo NI-DAQ USB-6008 para enviar señales del controlador al computador donde estaba modelada la planta virtual.

En la figura 8 se pueden observar las entradas en verde, que son de tipo booleanas, de acuerdo con el formato en que se accederá a las salidas del selector. También se puede observar en la misma figura las salidas en azul, las cuales están normalizadas a $5 \mathrm{~V}$ para poder ser adquiridas mediante el circuito de conversión analógico/digital. Nótese que se está tomando el valor de 35.000 como el equivalente a $5 \mathrm{~V}$, por lo cual en el código de lectura $\mathrm{ADC}$ se multiplicará la lectura por una constante que sea compatible con esta conversión:

$$
\mathrm{V}_{\text {demanda }}=\text { LecturaADC } \cdot \frac{35000}{4095}
$$

El valor de 4095 se debe a los 12 bits de resolución del ADS7841. 
Figura 8

Planta de molienda simulada en LabView

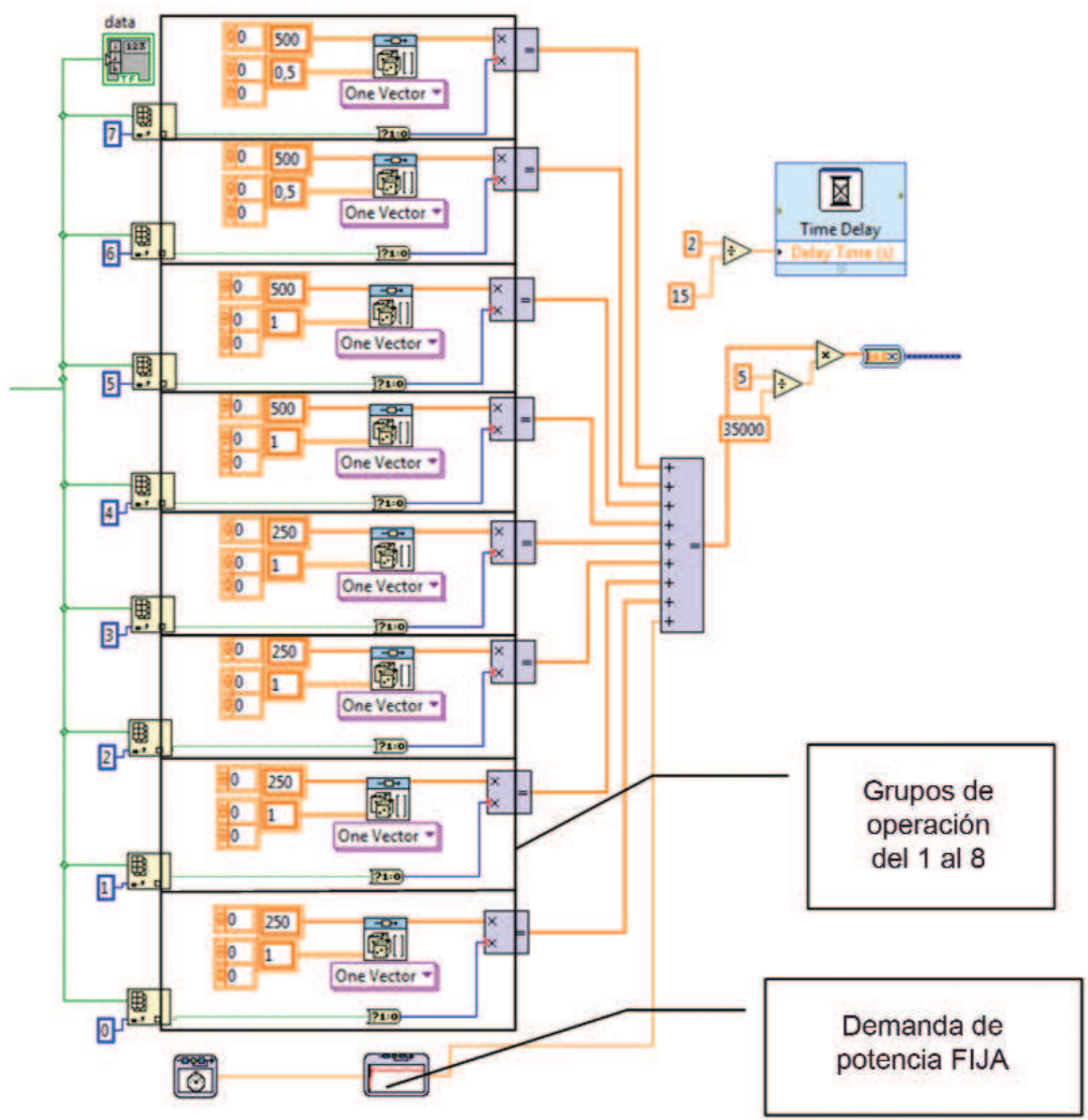

Elaboración propia. 
Enseguida se agregó a este diagrama las entradas y salidas de la $\mathrm{DAQ}$, como se muestra en la siguiente figura:

Figura 9

Planta con entradas y salidas desde y hacia la DAQ

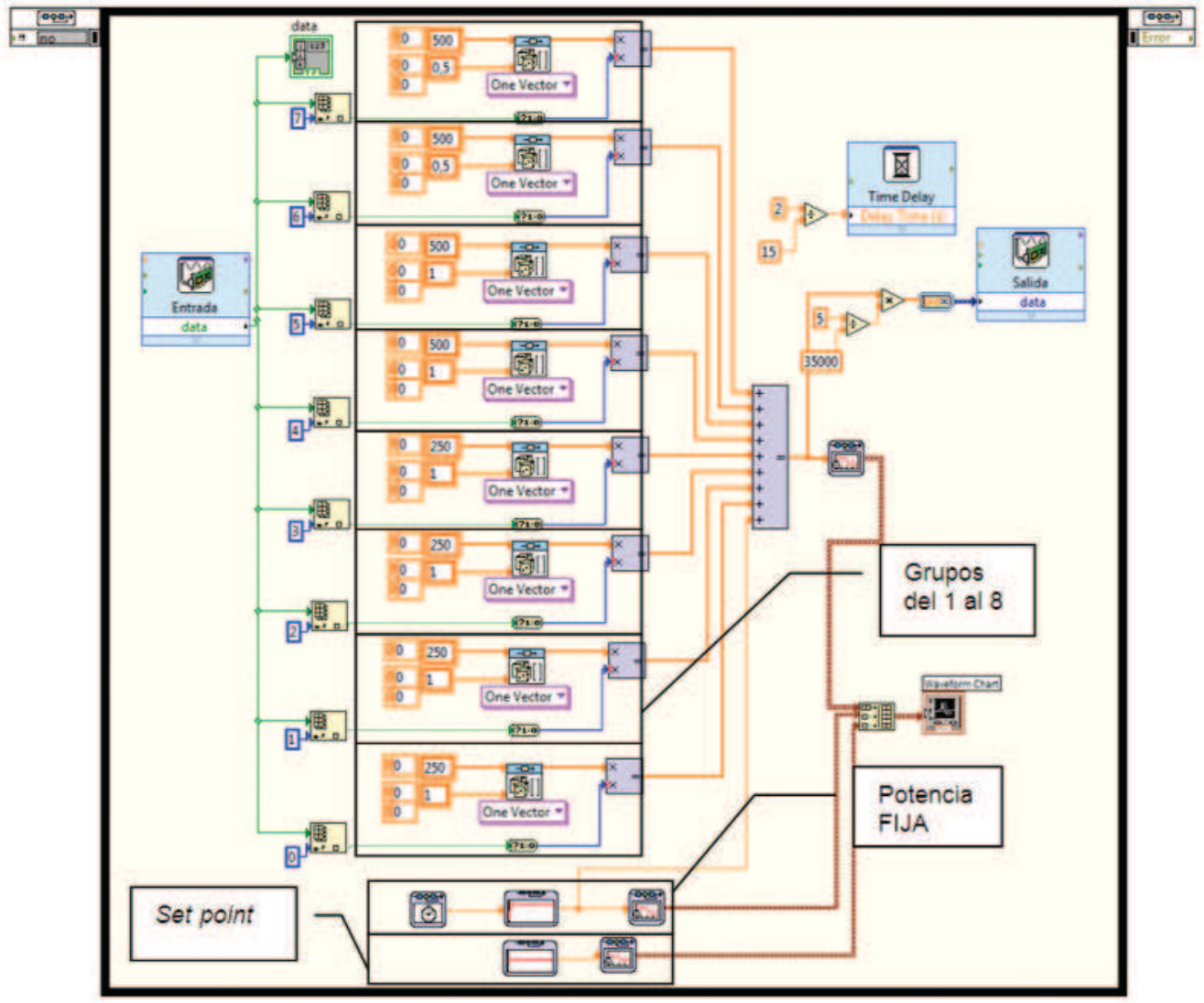

Fuente: Concentradora Toquepala.

Elaboración propia. 
En esta toma se observa cómo el controlador mantiene la curva de demanda (color blanco) por debajo de la demanda máxima contratada (línea verde). La curva de color rojo resalta el comportamiento de demanda de los equipos que necesariamente deben funcionar para no perjudicar el proceso productivo.

Figura 10

Controlador digital predictivo de máxima demanda

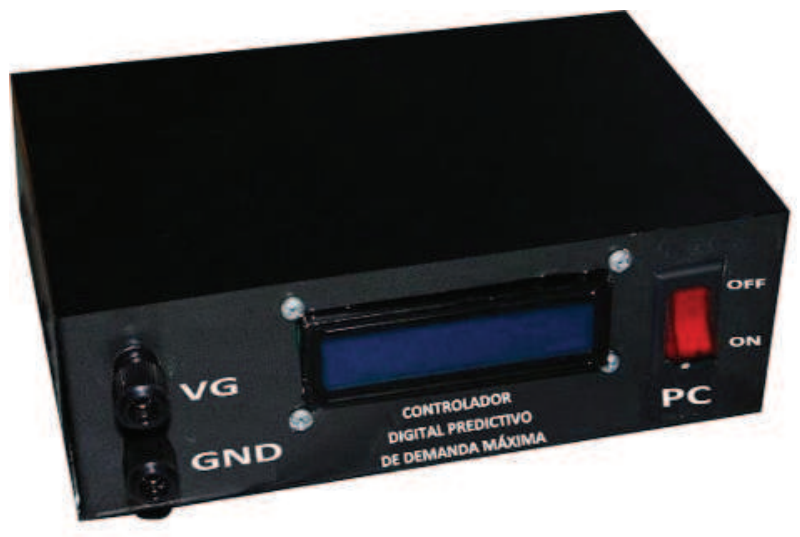

Figura 11

Funcionamiento del controlador en hardware

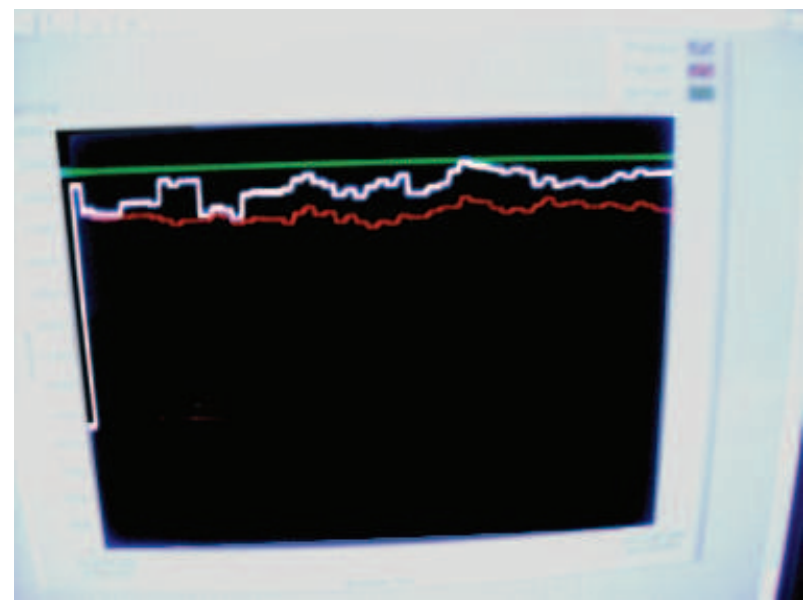

Elaboración propia. 


\section{DISCUSIÓN DE LOS RESULTADOS}

\subsection{Hallazgos más relevantes}

El formato "punto flotante" no es adecuado para trabajar con valores de variables muy altos y con muchos decimales; en cambio, se recomiendan las variables bajo el formato punto fijo. Asimismo, para desarrollos comerciales es conveniente hacerlo con procesadores de mejores características para el trabajo en condiciones difíciles de operación y con mejor manejo de grandes variables al realizar la implementación del controlador que laborará en planta.

\subsection{Posibles limitaciones metodológicas y razones por las cuales pueden ser válidos los resultados}

La metodología propuesta en esta investigación es lo suficientemente consistente para dar por válidos los resultados. La fase de experimentación se hizo sobre una planta virtual en LabView, que es la sección de molienda de la concentradora Toquepala, cuyo sistema de energía eléctrica, incluyendo el proceso productivo, se modeló matemáticamente en Matlab, que refleja con bastante precisión los indicadores más representativos del sistema real.

$\mathrm{Al}$ tener la planta virtual se simuló inicialmente el controlador en dicha planta, cuyos resultados comprobaron la eficacia del diseño, y luego se implementó el controlador en hardware; es decir, el dispositivo electrónico físicamente conectado a la planta virtual mediante un DAQ (conversor de señales).

Asimismo, los resultados de las pruebas han podido ser validados con la muestra elegida, que resulta ser suficientemente representativa para validar la eficacia del controlador de demanda máxima.

\subsection{Relación con publicaciones similares y análisis de similitudes y diferencias con hallazgos de otros autores}

En cuanto a las aplicaciones comerciales, se han analizado diversas propuestas para el control de demanda; entre ellas el modelo de control de demanda de Schneider Electric para eliminar picos de demanda con un control automático sincronizado en tiempo real, mediante el uso de algoritmos de demanda predictiva. Asimismo, se han revisado otras propuestas como las de ENth Energy y Sustanaible Energy 
Management y DL Steiners Electrical Engineers; pero ninguna ofrece información teórica acerca de sus modelos de control.

\subsection{Indicaciones y directrices para futuras investigaciones exponiendo nuevas hipótesis cuando esté justificado, etiquetándolas claramente como tales}

Actualmente, el controlador se ha trabajado con tarjetas de desarrollo; sin embargo, estas tarjetas, como su nombre lo indica, están orientadas al desarrollo y prueba de aplicaciones, por lo cual cuentan con elementos que muchas veces no son utilizados. Es conveniente diseñar e implementar una tarjeta que contenga únicamente los elementos necesarios para el funcionamiento del controlador, que permita reducir los costos de fabricación del dispositivo electrónico. Asimismo, estas tarjetas deben ser probadas en hardware para evaluar su idoneidad en aplicaciones industriales.

\section{CONCLUSIONES}

La conclusión inicial es que se ha podido probar la hipótesis de esta investigación, al verificarse en campo que el controlador digital predictivo de demanda, que se ha construido en este proyecto, mejora la gestión de demanda de potencia eléctrica, pues evita que se produzcan demandas no deseadas, según puede verse en el apartado 8.7 en la etapa de validación del controlador en hardware, que se hizo en la planta virtual de sección de molienda de la concentradora Toquepala.

La eficacia del dispositivo electrónico reside en su estrategia predictiva, pues el equipo es capaz de hacer una previsión de lo que va a suceder al final del periodo de 15 minutos y optimiza las cargas para tener la mayor cantidad de cargas conectadas posible, pero sin que exceda el límite máximo programado. Este pronóstico de demanda ha alcanzado un alto nivel de precisión, al llegar a un error menor al 1\%, conforme puede verse en el apartado 8.4.

Asimismo, se ha logrado optimizar las cargas que deben mantenerse en operación en las distintas franjas tarifarias mediante un modelo de programación lineal binaria, que ordena la secuencia de operación de grupos de equipos (véase el apartado 8.1). Este modelo puede simplificarse para dos tipos de tarifa, que resulta ser habitual en el mercado de electricidad: horas punta (18:00 a 23:00 horas) y 
fuera de punta durante el resto del día, y aplicarse a cualquier empresa comercial o de servicios.

Por otro lado, este controlador presenta un bajo costo de implementación y resulta comparativamente más barato que los equipos comerciales similares, al estar construido con tecnología propia e insumos mayormente nacionales; pero principalmente porque no requiere de hardware ni software especializados, conforme se aprecia en los apartados 8.2 y 8.3. Este tipo de tecnología, por su bajo costo, se puede extender masivamente a las mypes y reducir los sobrecostos de facturación en la pequeña y mediana empresa.

Este equipo, al eliminar los picos de demanda no deseados (véase el apartado 8.7) usa eficientemente la energía eléctrica, lográndose con ello un impacto beneficioso para el medio ambiente, pues la demanda de energía eléctrica en horas punta es cubierta principalmente con plantas térmicas, que operan con carbón o diésel, y son fuentes de emisiones de dióxido de carbono.

\section{BIBLIOGRAFÍA}

Bakirtzis, A. et al. (agosto de 1995). "Short term load forecasting using fuzzy neural networks". IEEE Transactions on Power Systems. Volume 10, Issue 3. Nueva Jersey: IEEE Power \& Energy Society.

Bertsekas, Dimitri (2000). Dynamic programming and optimal control.

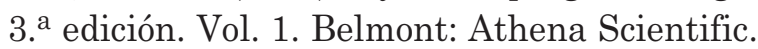

Brinkmann, Klaus (2002). Integration und management dezentraler energieversorgung. Modul ausgewählte aspekte des energiemanagement. Coblenza: Universität Koblenz.

Minem, Dirección General de Electricidad (2009). Guía $N^{\circ}$ 3: Elaboración de proyectos de guías de orientación del uso eficiente de la energía $y$ de diagnóstico energético - sector textil. Lima: Ministerio de Energía y Minas.

$\mathrm{EN}^{\text {th }}$ Energy. "Sustanaible energy management". <http://www. enthenergy.com/Energy/DemandControl/tabid/106/Default.aspx>. [Consulta: 13 de mayo del 2010.]

Hufendiek Kai und Kaltschmitt, Martin (1997). "Einsatz künstlicher neuronaler Netze bei der kurzfristigen last prognose". Estútgart: Institut für Energiewirtschaft und rationelle Energieanwendung. 
International Council on Large Electric Systems-CIGRE Working group WG 39.03. (diciembre de 1992). "Present practices on load forecasting and load management: A survey". Electra 145. París: International Council on Large Electric Systems-CIGRE.

Mallo González, Carlos (2002). "Predicción de la demanda eléctrica horaria mediante redes neuronales artificiales". Oviedo: Universidad de Oviedo, Departamento de Economía Cuantitativa.

Meisenbach, Christian (1999). "Lastprognose konventionell oder mit neuronalen Netzen - ein Vergleich". Optimierung in der Energieversorgung: Planungsaufgaben in liberalisierten Energiemärkten. Düsseldorf: VDI-Verlag.

Ministerio de Energía y Minas, Oficina de Planeamiento, Inversiones y Cooperación Internacional (2009). Balance nacional de energía 2008. Lima: Minem.

Radgen, Peter (2002). "Technik der Rationellen Energienutzung". Teil 1, Modul Energietechnik / Rationelle Energienutzung. Coblenza: Universität Koblenz.

Research \& Education Association (2004). Mechanical engineering handbook. Nueva Jersey: Research \& Education Association.

Sánchez, Edgar y Alma Alamis (2004). Predicción de la demanda eléctrica usando redes neuronales, entrenadas por filtro de Kalman. Michoacán: Universidad Michoacana de San Nicolás de Hidalgo.

Schneider Electric. "Control de demanda". <http://www.schneider-electric.com.mx>. [Consulta: 12 de abril del 2010.]

Siegelmann, Hava (abril de 1997). "Computational capabilities of recurrent NARX neural networks". IEEE Transactions on Systems, Man, and Cybernetics, Part B: Cybernetics. Volume 27, issue 2. Nueva York: IEEE Systems, Man, and Cybernetics Society.

Turabian, Kate L (2007). Manual for writers of research, papers, theses and dissertations: Chicago style for students and researchers. $3 .^{\mathrm{a}}$ edición. Chicago: University of Chicago Press.

Venero, Antony (2004). "A neural network approach to predict hurricane intensity in the north Atlantic basin". Tesis para optar el grado de Maestro en Ciencias en Ingeniería Industrial. Mayagüez: Universidad de Puerto Rico. 